MANCHESTER

SCHOOL OF

Computer Science

Information

Management Group

WEL- COPE Technical Report 3, October 2012

\title{
Coping Tactics Employed by Visually Disabled Users on the Web
}

\author{
Markel Vigo and Simon Harper \\ Web Ergonomics Lab \\ School of Computer Science \\ University of Manchester \\ UK
}

Interaction on the Web is often problematic for visually disabled users. In order to analyse how visually disabled users deal with problematic interactions we carried out a secondary analysis of 2 independent datasets containing the interaction of 25 users. As a result, we identified a number of problematic situations including uncertainty, reduced mobility, confusion and overload, and observed 17 tactics employed to overcome these situations, being impulsive clicking, exploration tactics and re-doing some of the most noteworthy tactics. These tactics are novel in that they are contextualised and complete: their presence denotes the presence of specific problems. Therefore these tactics are understood as behavioural markers of cognitive processes that indicate problematic interactions. We highlight the importance of these behavioural markers for designers and tools in order to detect and pre-empt problematic interactions. We also explore how we can remove the need to cope in these problematic situations.

Web Ergonomics Lab 


\section{COPE}

The aim of the COPE project is to investigate the behavioural strategies users adopt while interacting with the World Wide Web, identifying coping situations and providing technological solutions to overcome such difficulties.

\section{COPE Reports}

This report is in the series of WEL COPE technical reports. Other reports in this series may be found in our data repository, at http://wel.cs.manchester.ac.uk/research/cope/. Reports from other Web Ergonomics Lab projects are also available at http://wel-eprints.cs.manchester . ac.uk/.

\section{Acknowledgements}

COPE is funded by the Department of Education, Universities and Research of Basque Government, reference: BFI-2010-305.

\section{License}

This report is licensed under a Creative Commons Attribution-NonCommercialShareAlike 3.0 Unported License; see http://creativecommons.org/licenses/ by-nc-sa/3.0/. 


\section{Contents}

1 Introduction 1

2 Adaptive Behaviour and Coping $\quad 1$

3 Observational Setting and Data Acquisition 2

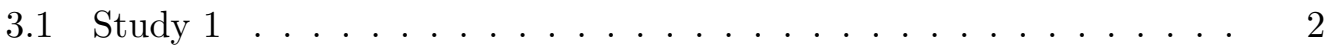

3.1 .1 Type of Data Analysed . . . . . . . . . . . . . . 3

3.1 .2 Users . . . . . . . . . . . . . . . . 3

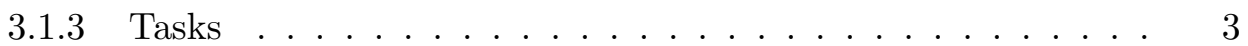

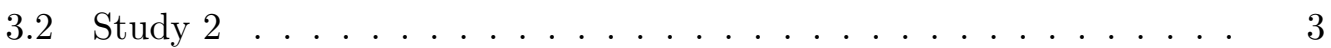

3.2 .1 Type of Data Analysed . . . . . . . . . . . . . 4

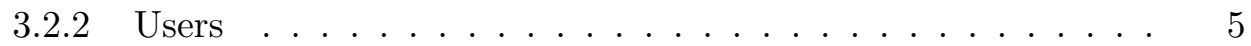

3.2 .3 Tasks . . . . . . . . . . . . . . . 6

3.3 Data Analysis Method . . . . . . . . . . . . . . . 6

4 Inventory of Problems Faced $\quad 7$

4.1 General coping cases . . . . . . . . . . . . . . . . 7

4.1 .1 Uncertainty . . . . . . . . . . . . . . . 7

4.1 .2 Reduced Mobility . . . . . . . . . . . . . 7

4.1 .3 Confusion . . . . . . . . . . . . . . . . . 8

4.1 .4 Overload . . . . . . . . . . . . . . . 8

4.2 Specific emergent coping situations . . . . . . . . . . . . 9

4.2.1 Accessibility and Usability (A \& U) . . . . . . . . . . . 9

4.2 .2 Unmet Expectations $(\mathrm{UM}) \ldots \ldots \ldots$

$4.2 .3 \quad$ Inter-page Navigation Problems $(\mathrm{N}) \ldots \ldots \ldots$

4.2.4 Intra-page Exploration Problems (E) . . . . . . . . . . . 9

4.2 .5 Information Architecture (IA) . . . . . . . . . . . . . 10

4.2 .6 Assistive Technology Issues (AT) . . . . . . . . . . . . 10

4.2 .7 Emotional Coping $(\mathrm{EM}) \ldots \ldots \ldots \ldots$

4.3 Mapping from general to emerging coping situations . . . . . . . . . 10

4.3 .1 Uncertainty . . . . . . . . . . . . . . . . . . . . 11

4.3 .2 Reduced mobility . . . . . . . . . . . . . . . 11

4.3 .3 Confusion . . . . . . . . . . . . . . . . . . . . 12

4.3 .4 Overload ........................... 13

5 Compendium of Tactics Employed $\quad 14$

5.1 Tactic 1: Asking for Assistance. . . . . . . . . . . . . . . . . . . . . . 14

5.2 T2: Impulsive Clicking. . . . . . . . . . . . . . . . . . . . 14

5.3 T3: Exploration Tactics. . . . . . . . . . . . . . . . 15

5.4 T4: Giving Up. . . . . . . . . . . . . . . . . . . . 16

5.5 T5: Gaining Orientation. . . . . . . . . . . . . . . 16

5.6 T6: Re-doing: Re-check, Re-type, Re-trace and Re-start. . . . . . . . 16

5.7 T7: Not Operating or Delegating on Assistive Technology. . . . . . . 17

5.8 T8: Trial \& Error. . . . . . . . . . . . . . . . . . . . . . . 17 
6 Discussion 19

6.1 On the Problems and Coping Situations Discovered . . . . . . . . . 19

6.2 On the Tactics Employed . . . . . . . . . . . . . . . . . 19

6.3 On the Relationship between Problematic Situations and Tactics Employed ........................... 20

6.4 Removing the Need to Cope . . . . . . . . . . . . . . . . . . . . 21

7 Conclusion $\quad 22$

8 Associated Files $\quad 25$

Web Ergonomics Lab

School of Computer Science

University of Manchester

Kilburn Building

Oxford Road

Manchester

M13 9PL

UK

tel: +44 1612757821

http://wel.cs.manchester.ac.uk/
Corresponding author:

Markel Vigo

tel: +44 1612757821

markel.vigo@manchester.ac.uk

http://www . markelvigo.info 


\section{Introduction}

In a previous technical report of the COPE project [19] we proposed a number of research actions for the advancement of Web interaction modeling. These actions are shaped as a research programme that sets a special focus on exploring the effects that problematic situations on the Web (i.e., coping) have on Web interaction. Therefore a particular attention is paid to the steps taken, the decisions made, the strategies employed and the situations that bring about such strategies. If we are able to identify the coping strategies exhibited by users in determined situations we will be able to (a) recognise coping behaviours as they occur in real-time, (b) transform the interface to adjust it to the needs of the user, (c) pre-empt these situations when they happen and (d) inform navigation models so that they consider coping behaviours.

Consequently, this report identifies the coping behaviours exhibited by visually disabled users on the Web. To do so, we conducted a secondary analysis of two independent datasets accounting for the interaction of 25 visually disabled users (see section 3). Using coping theory as a framework of reference (see section 2), we classify the problematic encounters into problems caused by uncertainty, reduced mobility, confusion and overload in section 4.1. In a second pass (see section 4.2) we identify the emerging situations including accessibility and usability, unmet expectations and information architecture that generate coping behaviours. In order to explore the specific cases of the general case we provide a mapping between a Web technology driven classification and general coping situations in section 4.3. By proceeding in this way we suggest the removal of general coping situations from a technical perspective. Then, in section 5, we analyse how users react and what are the solutions and workarounds employed (namely tactics) in order to overcome or bypass such problems. We analyse the problematic situations identified with regard to previous work in section 6; we also contextualise tactics in terms of coping tactics and we discuss how by relating coping situations to the tactics employed paves the way to programmatically detect problematic situations and automatically adapt the interface. As a result, we finally propose a number of techniques to remove the need to cope.

\section{Adaptive Behaviour and Coping}

As we mentioned in the first report of the COPE project [18], most of the time individuals operate consistently in an environment solving problems by applying habitual mechanisms and reacting in a predictable way. When these mechanisms do not work, the arising tension produces frustration, anxiety and helplessness. Under these circumstances adaptation works as an acceptable compromise with the environment rather than a triumph or a total surrender over it. Adaptive behaviour, and especially coping are tools to overcome these problematic situations. Coping is defined as the "constantly changing cognitive and behavioural efforts to manage specific external or internal demands that are appraised as taxing or exceeding the resources of a person" [9]. Coping is about responding to external situation requirements and responding to the threat posed by such situations; therefore, coping is 
understood as an extreme adaptation. Note that coping is an adaptive behaviour but not all adaptive behaviours are coping. Appraisal is the evaluative stage that precedes coping which is defined as the individuals' evaluation of environmental stimuli. There are some situational factors at the appraisal stage that make the encounters potentially threatening: situations in which individuals are unfamiliar with the setting cause uncertainty, confusion is caused by the lack of environmental cues that lead to discover and learn about a situation that will happen. Additionally, the confusion caused by the environmental configuration and the lack of situational clarity do also lead to coping situations. Typically, the goal of the actions taken is to overcome the troublesome situation [21]; to do so, individuals employ tactics to manage the following variables:

- By keeping the adequate information amount about the environment, the cognitive field of individuals has the right amount of information as a guide to action. When the information is scarce, individuals make the most of the existing little information or seek for more information. Conversely, if the amount of information is overwhelming, attentional strategies such as selective attention help to reduce information overload.

- Maintaining satisfactory internal conditions in terms of information processing enables to be ready for any event that may happen.

- Maintaining autonomy or freedom on the environment requires a constant monitoring of the available escape ways that lead to an equilibrium situation. When individuals are deprived from autonomy or freedom, behaviour strategies aim at maximizing the existing little operational capacity.

If the employed tactics allow to overcome the problematic situation, it can be said that the individual has adapted to the situation. For a full discussion about adaptive behaviour and coping on the Web see the first report of the COPE series $[18]$.

\section{Observational Setting and Data Acquisition}

In order to expand on the hints provided by previous work, we conducted a secondary analysis of two datasets and transcriptions belonging to two independent studies, where data were collected by unobtrusive observation. Different investigators ran each study allowing the removal of the possible bias introduced. When analysing data to identify coping situations and behaviours, in situ observations have a number of benefits compared to other data collection methods: In situ observations allow to recognise emergent behaviours, identify the sources of user frustration and enable a better understanding of why users employ determined tactics.

\subsection{Study 1}

The goal of this study was to analyse the interaction of blind and visually impaired users on the Web. A think-aloud method was employed so that the observer could record what users verbalised jointly with interaction data. The datasets can be found in [11]. 


\subsubsection{Type of Data Analysed}

As mentioned, the dataset not only contains the verbalisations of the user that correspond to the think-aloud protocol but also there are additional explanatory comments made by the observer that clarify the situations; the interaction with the keyboard and mouse, and the commands executed are also recorded. An excerpt of the interaction of $u 13$ in this dataset looks as follows ${ }^{1}$ :

- Arrow Down to select Africa [by mistake]

- [Page brings up Telegraph Travel window but JAWS does read it out]

- (It's gone, the information refuses to come up)

- Click on back (I'll start again)

- Read out top of the page [user is in the travel page, not in the weather page but he does not realise]

- Tab down through links

- Tab up page (where is the weather?)

\subsubsection{Users}

Table 1 shows the profile of the 20 participants that took part in the study: 13 blind users (65\%), 6 visually impaired (30\%) and 1 dyslexic user (5\%). 16 of them (80\%) used screen readers in the experiment (2 of them used it jointly with a Braille display) distributed as 16 JAWS users (69\%), 3 SuperNova users (19\%) and the remaining 2 , who accounted for a $13 \%$, used another screen reader. 1 visually impaired user used a screen magnifier, while another visually impaired user used the screen reader in addition to the screen magnifier. Finally, 2 visually impaired users did not use any assistive technology at all but just looked closer to the screen. All participants had more than 5 years of experience in the Web so we considered them to be expert users.

It can be observed that the group was not homogeneous and the usage of assistive technologies varied across users and disabilities. Unsurprisingly, some visually impaired users employed screen readers, while some other did not use any assistive technology. A sighted dyslexic user also made use of the screen reader as a way to better understand the text of the sites.

\subsubsection{Tasks}

Each has to accomplish 4 tasks without any time limitation; they had to find (1) the weather forecast for a specific location, (2) specific information about a journal in a digital library, (3) information about Salomon Islands in a large catalogue of resources, and (4) purchase a suit in a large department store.

\subsection{Study 2}

The study presented in this report is based on in-situ observations of visually impaired users interacting with Web technology. These observations took place at the

\footnotetext{
${ }^{1}$ The square brackets are the comments made by the observer and the round brackets the verbalisation of the user.
} 
Table 1: Users taking part in study 1.

\begin{tabular}{|c|c|c|c|c|}
\hline id & $\begin{array}{l}\text { nature of im- } \\
\text { pairment }\end{array}$ & assistive technology & specific AT & experience \\
\hline$u 1$ & blind & screen reader & SuperNova & expert \\
\hline u2 & blind & screen reader & JAWS & expert \\
\hline u3 & blind & screen reader & SuperNova & expert \\
\hline$u 4$ & visually impaired & none & $\begin{array}{l}\text { User looks closer } \\
\text { to the screen }\end{array}$ & expert \\
\hline 45 & blind & screen reader & JAWS & expert \\
\hline$u 6$ & dyslexic & screen reader & JAWS & expert \\
\hline$u 7$ & blind & screen reader & JAWS & expert \\
\hline 48 & blind & screen reader & $\begin{array}{l}\text { JAWS and } \\
\text { Braille display }\end{array}$ & expert \\
\hline$u 9$ & visually impaired & screen reader & PW Webspeak & expert \\
\hline$u 10$ & blind & screen reader & JAWS & expert \\
\hline$u 11$ & visually impaired & screen reader & SuperNova & expert \\
\hline u12 & visually impaired & none & $\begin{array}{l}\text { User looks closer } \\
\text { to the screen }\end{array}$ & expert \\
\hline$u 13$ & blind & screen reader & JAWS & expert \\
\hline u14 & blind & screen reader & JAWS & expert \\
\hline$u 15$ & visually impaired & screen magnifier & Zoomtext & expert \\
\hline$u 16$ & blind & screen reader & HAL & expert \\
\hline$u 17$ & blind & screen reader & JAWS & expert \\
\hline$u 18$ & visually impaired & $\begin{array}{l}\text { screen reader and screen } \\
\text { magnifier }\end{array}$ & $\begin{array}{l}\text { JAWS and } \\
\text { Zoomtext }\end{array}$ & expert \\
\hline$u 19$ & blind & screen reader & JAWS & expert \\
\hline u20 & blind & screen reader & $\begin{array}{l}\text { JAWS and } \\
\text { Braille display }\end{array}$ & expert \\
\hline
\end{tabular}

Henshaw's Society for Blind People, during the Skillstep to Success course, where people with visual impairments learn to use technology and they are especially helped to develop Web access skills. Participants are taught all the basics about computers, Internet, browsers, screen reader and screen magnifier use so it can be understood as a course to acquire computer literacy. Users often talked among themselves in order to recommend a certain website or to give some assistance when they needed help. They were supervised by visually disabled tutors who gave them a crash course on basic commands before they were started. Sometimes, the tutors would also suggest some websites for job seeking purposes. This setting is invaluable because it allows to observe how the skill acquisition and learning process comes about and how strategies evolve over time. Transcripts and diaries of the sessions, interviews with users, the websites accessed, tasks conducted, problems encountered and the strategies adopted have been extracted from the SADIe series technical reports (see [12] and [13]).

\subsubsection{Type of Data Analysed}

The dataset contains a detailed description of the actions taken by users, how they employ assistive technology and the problems encountered. As an example, the following excerpt shows how user interaction is described in these datasets: "... the 
Table 2: Users taking part in study 2.

\begin{tabular}{llllll}
\hline id & $\begin{array}{l}\# \text { of } \\
\text { sessions }\end{array}$ & $\begin{array}{l}\text { nature of im- } \\
\text { pairment }\end{array}$ & assistive technology & specific AT & experience \\
\hline u21 & 5 & visually impaired & screen magnifier & Zoomtext & beginner \\
422 & 5 & visually impaired & $\begin{array}{l}\text { screen reader or screen } \\
\text { magnifier }\end{array}$ & $\begin{array}{l}\text { JAWS or Zoom- } \\
\text { beginner }\end{array}$ \\
u23 & 4 & blind & screen reader & JAWS & beginner \\
u24 & 2 & visually impaired & screen reader & JAWS & beginner \\
u25 & 2 & visually impaired & screen magnifier & Zoomtext & intermediate \\
\hline
\end{tabular}

button was not within u21's field of view due to screen magnification. Also, the background colour and button colour did not have a high contrast and u21 found it difficult to identify the button and click on it. It only when the mouse pointer changed from a pointer icon to a hand icon that ...".

\subsubsection{Users}

5 users were observed in periods of time comprising a maximum of 2 months. The small amount of number of users can be counterbalanced with long run multi-session observations, where a wide variety of behaviours can be exhibited; moreover, it helps to observe and detect not only usual navigational and interaction patterns, but also critical and outlying events that prevent peak experience.

Table 2 shows the profile of the participants: $u 21$ is visually impaired and makes use of Zoomtext. He had used the Internet before but when the course started he had already forgotten how to use it. 422 is visually impaired although his vision is variable. Some days he can see better than others and on these occasions he will make use of the Zoomtext screen magnifier. On a bad day the Jaws screen reader will be used. He has basic knowledge on computers although he is inexperienced on the Web. u23 is profoundly blind, has a guide dog and is a Braille competent user; his interaction method is the Jaws screen reader. He never used a computer prior to the course. u24 is visually impaired but not profoundly blind; he never used a computer before and spent most of his time learning basic computer functionalities. Only at the end of the course was able to start browsing using the Jaws screen reader. u25 is a experienced user who is visually impaired and uses the Zoomtext screen magnifier. His goal in the course was to acquire further browsing skills. Based on their skills and knowledge on accessing the Web, we can consider that all the subjects that took part in sudy 2 but 425 were mostly inexpert and unskilled.

Observation Period. u21 was observed in a period shorter than two months: 9/5/2007, 6/6/2007, 13/6/2007, 20/6/2007 and 27/6/2007. u22 was observed on $16 / 5 / 2007,23 / 5 / 2007,13 / 6 / 2007,20 / 6 / 2007$ and $27 / 6 / 2007$ comprising a period one month and a half; u23 was observed four times in a period of one month: on $7 / 11 / 2007,14 / 11 / 2007,5 / 12 / 2007$ and on 12/12/2007. u24 and u25 were observed in $13 / 06 / 2007$ and $27 / 06 / 2007$ respectively. 


\subsubsection{Tasks}

There were no structured tasks to accomplish as users were free to browse the Web. Users tended to browse on those sites they thought were necessary in their near future. For instance, they emulated the booking of a flight or the purchase of an item in an e-commerce site. Users also tried to browse on nationwide and local media; they also browsed for fun and this sometimes led them to serendipitous findings. However, they were encouraged by tutors to navigate through pages that would allow them to get a job as one of the goals of this course was to increase their employability prospects. Users were not constrained by time in any of their tasks. Those tasks that were interrupted because the class had reached its ending have not been considered in this analysis.

\subsection{Data Analysis Method}

We analysed the observational reports of study 1 and study 2 following the coding procedures of Grounded Theory [6] in a incident-to-incident fashion. This means that we did the coding in granularity level that allowed us to identify high level problematic events. In following the principles of Grounded Theory we aimed at identifying the emerging categories and entities instead of formulating a theory, proceeding as follows:

- Initially we did a first pass using categorical coding based on previous work on coping in the human and animal domain, which is built on solid foundations and experimental observations from the fields of ethology, biology or cognitive science (see section 2). By using general scope theoretical frameworks of coping, we are able to identify and describe the problems faced by users on the Web independently of how the underlying technology is deployed (see section 4.1). One of the weaknesses of current literature is that strategies employed by users are dependent on the implementation and design of web pages. For instance, one of the strategies found in [22] was that users counted the number of tabs in order to skip content at the top of the Google results page. If the mentioned web page updates, if the design patterns of websites evolve or if assistive technology provides the mechanisms to bypass such problems the coping strategy becomes obsolete. By employing a priori coding, we aim at having generalisable outcomes that are applicable in the future regardless the evolution of design patterns, Web technology or user agents. As a result we identified uncertainty, reduced mobility, confusion and overload as the main problems faced by visually disabled users on the Web.

- In a second pass we coded the data in a open or emergent fashion. In this way, the categories - in terms of problematic interactions - emerged without any intentional prejudice. As a result we came up with the following categories that determine problematic interactions on the Web: accessibility and usability, unmet expectations, navigation strategy, exploration strategy, information architecture, assistive technology and emotional issues (see section 4.2 for further information). 
- Finally, we mapped the general categories found as a result of employing categorical coding into the categories encountered by employing emergent coding. While the former categories are independent of the underlying Web technology, the latter are dependent on it. By proceeding in this way, we were able to identify the emergent specific cases of the general case (see section 4.3);

\section{Inventory of Problems Faced}

\subsection{General coping cases}

Coding revealed 133 troublesome situations in which the 25 users faced problems of a different nature. Bearing in mind existing theoretical frameworks on coping, we discovered the following categories that characterise coping in the Web: uncertainty, reduced mobility, confusion and overload. The boundaries of such categories were not always clear as sometimes we could find situations where more than one category occurred. For instance it was common to find confusing and overloading situations where each category appeared in a different degree. We decided to classify such situation based on its most prominent characteristic - especially if it was indicated by the user -, for instance: if confusion prevailed over being overwhelmed the event was classified as a situation of "confusion". When looking at the data, we found striking that experts experienced 85 problematic events ( $64 \%$ of problems), while beginners ( $20 \%$ of users) went through 48 ( $36 \%$ of problems). This can be explained in that the multi-session study may counterbalance the reduced number of inexperienced users and because novel users may face more problematic situations than skilled users.

\subsubsection{Uncertainty}

Previous research on coping with uncertainty [10] defines uncertainty as "the sense of doubt that blocks or delays the action" and establishes 3 situations that generate uncertainty: inadequate understanding, incomplete information and undifferentiated alternatives. The strategies adopted for coping with uncertainty are reducing uncertainty, assumption based reasoning, weighting pros and cons of competing alternatives and forestalling. We identified 26 events (20\% of the events) where individuals faced situations of uncertainty; these situations were common to 10 users (40\% of users). Unexpected banners, previous negative experiences in navigating through similar content or lack of alternative text for pictures are some of the problems that cause uncertain situations. u11 expressed uncertainty as "I can't read this but I will give a try, I am assuming it is what I want so let's see where it takes me"; also while $u 19$ was exploring a page and was not able to find what he was looking for, commented "I'm not confident this is what I want", when coming across some pictures that did not have any alternative text.

\subsubsection{Reduced Mobility}

Case studies describing the way in which concentration camp prisoners, prisoners of war or people who have suffered natural catastrophes behave give some hints about the coping strategies that are adopted in analogous situations where freedom and 
autonomy are reduced or non-existent [15]. The tactics adopted in such situations, amongst many others are: null coping, surrendering to coping, taking advantage of smallest portion of autonomy, focusing on small gratifications, etc. We identified 23 events $(17 \%)$ where users got stuck, disoriented, trapped or faced a extremely difficult interaction; 11 different users (44\%) underwent these sort of situations. Looping on a sequence of pages and not being able to escape, finding oneself in a dead-end web page or software compatibility problems depict some of these situations. These situations are illustrated by $u 11$ when realising he had unintentionally looped "I've got back to shorts again ... shorts again!" or u22, when he was not able to get out of the navigation bar; the observer comments "JAWS seemed to read the links many times, as though in an infinite loop". Some expressions illustrate how users got disoriented: "Oh, I've got lost, I don't know where I am now" as a consequence of $u 18$ clicking in the wrong link or "Not sure where I am ... if in doubt go back to the beginning", after u2 read all the results provided by a search engine.

\subsubsection{Confusion}

Confusion is a category built on the appraisal factors of novelty, unfamiliarity and ambiguity of the setting; therefore, the initial expectations of the user play a central role. According to our coding, when any of these factors is present confusion is likely to happen. Confusion was the category that accounted for the higher number of problematic experiences: 22 users (88\%) underwent confusing and ambiguous situations. A total number of 61 of these situations were identified (46\%). There is a broad scope of situations that generate confusion to users: problems with text size, the lack of search results, encountering unfamiliar content and functionalities, or misuse of assistive technology to name a few. Some examples of confusing situations are the one faced by $u 17$ when a search engine provided 0 results: "Where have the results gone?"; u20 was unable to distinguish between a link and text, "It's not there ... it's annoying me now ... it's not a link!".

\subsubsection{Overload}

Selective attention allows individuals to discriminate between many sources of information (through the visual and auditory channel) and select just the one it is targeted. It is a mechanism to overcome information overload by tracking some stimuli at the expense of others. This way, one can attend to what is important in a given environment and to adaptively cope with changed circumstances. When it comes to coping strategies, filtering and withdrawal emerge as the ones employed by individuals [17]. We found 11 users experiencing situations of overload (44\%) across 29 events (22\%). Some of the situations that illustrate overwhelming interactions are too many search results, too many steps to complete a transaction and high number of items in navigation menus. Overload can be illustrated by the following examples: when the prospect of a large amount of links deterred $u 8$ from carrying on: "900 links ... OK that's enough"; ur was aware that the page contained what he was looking for but the goal was buried somewhere in between all the information provided: "This is a problem sometimes, you can have 30 or 230 links that you have to sit and listen to!". 


\subsection{Specific emergent coping situations}

\subsubsection{Accessibility and Usability (A \& U)}

As expected there are a number of accessibility and usability problems that cause problems in the interaction of visually disabled users. While this category encompasses those problems that could be covered by accessibility guidelines [3], it can be argued that the remaining categories contain also accessibility and usability issues as they hinder the interaction of visually disabled users. Surprisingly accessibility and usability problems accounted for 24 problematic interactions (18\% of problems found) that were experienced by 8 users ( $32 \%$ of the participants). For instance when encountering pictures without alternative content $u 13$ described "[screen reader] keeps reading out part of weather page: 'min', 'min', 'min' "; in the case of u2, when a number of links were similarly labelled: "everything seems to say 'suit pieces', will probably have to click on every link to find out what happens".

\subsubsection{Unmet Expectations (UM)}

According to our analysis expectations of users tend not to be met due to a number of reasons: coming across unfamiliar layouts and functionalities, bumping into banners and advertisements and lack of results when querying search engines, etc. Unmet expectations account for $16 \%$ of problems (21 issues) and were experienced by 12 users accounting for $48 \%$ of the participants. For instance, $u 11$ got surprised when finding a banner about mortgages while searching for the weather forecast: "Mortgages? What's that got to do with the weather?"; similarly when no results are provided by a search engine $u 1$ expressed "Don't understand what all this is about, I'm giving up".

\subsubsection{Inter-page Navigation Problems (N)}

Inter-page navigation refers to the steps taken by users to reach to the page that contains their goal. Therefore, we focus on the hyperlink assessment made by users and the sequence of hyperlinks scanned and clicked. 19 problematic situations (14\%) were experienced by 14 users $(56 \%)$ due to problems in the navigation strategy adopted. Navigation problems can be illustrated as when $u 18$ realised he had looped while searching for information "Damn, I'm just going round in circles, I give up!"; when $u 13$ had wrongly implemented the backtracking technique (as he unintentionally skipped the page he was looking for) and got to another different one: "Where is the weather? ... I have come to the conclusion that I am on the wrong page".

\subsubsection{Intra-page Exploration Problems (E)}

Exploration strategy refers to the navigation of the user within a web page, it is all about reading and listening to content, scanning through links in order to find the goal or the link which will lead to the goal. The exploration strategy followed by users caused 18 troublesome interactions (14\%) to 13 users (52\%). Exploration problems can be described as the situation faced by $u 19$ when arrowing around (using the virtual cursor) deviated him from goal, which was very close to him, "I' $m$ 
going to give up on that". Another example of exploration problems is depicted by $u 11$ when he got to a page containing his goal but he missed the goal as he just gave a quick overview to the page without traversing it.

\subsubsection{Information Architecture (IA)}

This category refers to those elements of the interface that cause problems to users even if they are accessible or usable. They can be considered design problems as they are closely related to current website conventions in terms of layout and content arrangement (mast header, lateral navigation links menu, banner, content, footer, etc.) and the amount of information in each component. 10 users accounting for $40 \%$ of the participants experienced web interaction problems caused by information architecture issues for a total of 30 events (23\%). Such problems can be described as follows: u24 found too much textual information between the top and the main content of the page, "This is doing my head in this". Similarly, a number of links sat in between the location of 42 and his desired content: "There are lots of links between the search box and results".

\subsubsection{Assistive Technology Issues (AT)}

There are some interaction problems that are caused by the lack of knowledge about specific features of screen readers, misuse of their functionalities of because users forget to activate a specific interaction mode (i.e., forms mode in JAWS). 7 issues (5\%) of this type were found, affecting to 5 users (20\%). For instance, a software compatibility problem frustrated $u 16$ : "this is a waste of time ... it just will not read it ... don't want this ... can't go on this ... can't understand it"; the lack of synchronisation between the screen magnifier and the screen reader lead $u 18$ to an unwanted location: "I meant to click on search [link]".

\subsubsection{Emotional Coping (EM)}

This category refers to the events that are constrained by a past experience in a similar page or in the previous task. The current mood and the confidence on what users are doing plays a key role in determining users' interaction on the Web. These events are scarce as they account for the $6 \%$ of situations ( 8 encounters) and are experienced by 5 users (20\%). As an example of emotional coping, u21 was disheartened because of a previous bad experience, influencing negatively his subsequent experience. Similarly, after unsatisfactorily exploring the page $u 19$ clicked on a link not being sure of what he was doing "I'm not confident this is what I want".

\subsection{Mapping from general to emerging coping situations}

This section describes how emerging categories described in section 4.2 are related to general coping categories found as a result of a priori coding (see section 4.1). Therefore problems experienced by users are described within each emerging category in the scope of general coping categories. Table 3 shows the number of users 
Table 3: Mapping between general to emerging coping situations: number of users experiencing problems

\begin{tabular}{l|l|l|l|l|l|l|l|l}
\hline & $\begin{array}{l}\text { distinct } \\
\text { users }\end{array}$ & A\&U & UM & N & E & IA & AT & EM \\
\hline uncertainty & $10(40 \%)$ & 6 & 4 & $\mathrm{n} / \mathrm{a}$ & $\mathrm{n} / \mathrm{a}$ & $\mathrm{n} / \mathrm{a}$ & $\mathrm{n} / \mathrm{a}$ & 5 \\
reduced mobility & $11(44 \%)$ & 3 & $\mathrm{n} / \mathrm{a}$ & 7 & 4 & 1 & 3 & $\mathrm{n} / \mathrm{a}$ \\
confusion & $22(88 \%)$ & 2 & 12 & 12 & 13 & $\mathrm{n} / \mathrm{a}$ & 3 & $\mathrm{n} / \mathrm{a}$ \\
overload & $11(44 \%)$ & $\mathrm{n} / \mathrm{a}$ & $\mathrm{n} / \mathrm{a}$ & 1 & $\mathrm{n} / \mathrm{a}$ & 11 & $\mathrm{n} / \mathrm{a}$ & $\mathrm{n} / \mathrm{a}$ \\
\hline
\end{tabular}

Table 4: Mapping between general to emerging coping situations: number of problematic interactions

\begin{tabular}{l|l|l|l|l|l|l|l|l}
\hline & $\begin{array}{l}\text { total \# } \\
\text { issues }\end{array}$ & A\&U & UM & N & $\mathbf{E}$ & IA & AT & EM \\
\hline uncertainty & $26(20 \%)$ & 14 & 4 & $\mathrm{n} / \mathrm{a}$ & $\mathrm{n} / \mathrm{a}$ & $\mathrm{n} / \mathrm{a}$ & $\mathrm{n} / \mathrm{a}$ & 8 \\
reduced mobility & $23(17 \%)$ & 4 & $\mathrm{n} / \mathrm{a}$ & 9 & 5 & 2 & 3 & $\mathrm{n} / \mathrm{a}$ \\
confusion & $61(46 \%)$ & 6 & 17 & 14 & 13 & $\mathrm{n} / \mathrm{a}$ & 4 & $\mathrm{n} / \mathrm{a}$ \\
overload & $29(22 \%)$ & $\mathrm{n} / \mathrm{a}$ & $\mathrm{n} / \mathrm{a}$ & 1 & $\mathrm{n} / \mathrm{a}$ & 28 & $\mathrm{n} / \mathrm{a}$ & $\mathrm{n} / \mathrm{a}$ \\
\hline
\end{tabular}

that experienced problematic interactions at each category, whereas table 4 depicts the number of events that were found.

\subsubsection{Uncertainty}

\section{Accessibility and usability problems.}

- A number of pictures without alt text (2 users, 3 issues).

- Table layout renders text oddly (2 users, 5 issues).

- Problems with text size and contrast (1 users, 3 issues).

- No proper labelling for links (2 users, 2 issues).

- Horizontal scrolling is needed to reach a key functionality (1 users, 1 issue).

\section{Unmet expectations.}

- No search results (1 user, 1 issue).

- Unfamiliar layout of accessible pictures (1 user, 1 issue).

- Banner (2 users, 2 issues).

\section{Emotional coping.}

- Disheartened due to previous experience as user actions did not give expected outcomes (2 users, 2 issues).

- Losing confidence on what the user is doing (4 users, 6 issues).

\subsubsection{Reduced mobility}

\section{Accessibility and usability problems.}

- Lack of support for global orientation (1 user, 1 issue).

- Too much information between user's current location and desired content (1 user, 2 issues). 
- There are a number of linking pictures without alt text (1 user, 1 issue).

\section{Navigation strategy.}

- Bad navigation strategy, clicking on the wrong link (3 users, 3 issues).

- Lack of support for global orientation (1 user, 1 issue).

- Clicking on a link did not change the page, as the user was already in that page (1 user, 1 issue).

- Mistakenly clicking on a link and ending up on dead end (1 user, 1 issue).

- User trapped on a loop of pages as not obvious escaping ways and links towards the goal can be found ( 3 users, 3 issues).

\section{Exploration strategy.}

- Difficult exploration as the page is full of obstacles (1 user, 1 issue).

- Use trapped on a loop within a web page (1 user, 1 issue).

- Navigation style using screen magnifier (1 user, 2 issues).

- User gets lost after reading a list of unsatisfactory search engine results (1 user, 1 issue).

\section{Information architecture}

- Too many links and drop-down menus (1 user, 2 issues).

\section{Assistive technology issues.}

- Uncompatible browser does not work on page (2 users, 2 issues).

- User does not know how to use certain functionality of the screen reader (1 user, 1 issue).

\subsubsection{Confusion}

\section{Accessibility and usability problems.}

- Lack of support for global orientation (1 user, 1 issue).

- User is on the goal page but the images contain the key information are not accessible so the user is unable to know whether he is on the right place (1 user, 2 issues).

- Poor page layout confuses site and page links (1 user, 2 issues).

- Disappearing dynamic content in a carousel (1 user, 1 issue).

\section{Unmet expectations.}

- No search results (1 user, 1 issue).

- Banner (1 user, 1 issue).

- Unfamiliar functionality (1 user, 2 issues).

- Unfamiliar layout and contents arrangement (2 users, 3 issues).

- User is not able to ascertain whether the page he is exploring contains the goal (1 user, 2 issues).

- User is unaware the he has found the goal of the task (1 user, 1 issue).

- After performing a search, results are not right after but after a number of links (1 user, 1 issue).

- Unexpected character (1 user, 1 issue).

- User expects a piece of text, which is closely related to the goal, to be a link and keeps on unsuccessfully clicking on it (5 users, 5 issues). 


\section{Navigation strategy.}

- User confused as a consequence of clicking on low-scented links (6 users, 7 issues).

- Badly implemented backtracking (1 user, 1 issue).

- User eventually deviates from the good traversal path; he decides to loop in order to find the turning point (1 user, 1 issue).

- User realises he has looped (1 user, 1 issue).

- User clicks on highly scented links that will not lead him to the goal: high quality distractors (4 users, 4 issues).

\section{Exploration strategy.}

- Non accurate keyword for search engine (3 users, 4 issues).

- User is on the goal page but he gives a fast overview to the page and missing the goal as a consequence (4 users, 4 issues).

- Users go so fast through links that they miss the one that will lead them to the goal (2 users, 2 issues).

- Arrowing around with the virtual cursor deviates user from the goal which is located quite close to from his current location (1 user, 1 issue).

- Key functionality (search button) is out the field of view due to the user's exploration style (1 user, 1 issue).

- Unintentional mistakes made when clicking on long list of links (4 users, 4 issues).

\section{Assistive technology issues.}

- Misuse of assistive technology or the user forgot to activate its functionalities (2 users, 3 issues).

- Lack of synchronisation between screen reader and screen magnifier (1 user, 1 issue).

\subsubsection{Overload}

\section{Information architecture.}

- Too many search results (4 users, 4 issues).

- Too many links (2 users, 2 issues).

- A familiar and known link is present in a web page with a high amount of links (1 user, 1 issue).

- The user has the certainty that the link he is looking for is there but there is a high amount of links (3 users, 3 issues).

- The user has the certainty that the link he is looking for is within a very long list of items which are ordered according to some criteria (alphabetically, per year, etc.) (5 users, 5 issues).

- User expects cluttered content (2 users, 5 issues).

- Navigation elements are read time and again (1 user, 1 issue).

- Unnecessary information arranged in a highly sequential fashion (2 users, 2 issues).

- Too much textual information between user's location and desired content of the page (4 users, 4 issues). 
- Too many screens to traverse and forms to fill out in order to complete a process (1 user, 1 issue).

\section{Navigation strategy.}

- Use of suboptimal technique to navigate through the links of a page (1 user, 1 issue).

\section{Compendium of Tactics Employed}

When facing the mentioned problematic situations users employ different tactics. However, there is not a unique way of implementing each tactic but there are several ways of doing it. As a result we describe the tactics and the possible implementations we have observed; table 5 shows details about the frequencies and number of users that employed each tactic and their implementations.

\subsection{Tactic 1: Asking for Assistance.}

7 users asked for assistance 15 times for many different purposes and in diverse contexts of use. Active invoke for help was described as one of the main coping mechanism employed by individuals in real life [4]. In our analysis, $28 \%$ of users employed this tactic when encountering problems; the tactic was especially used by those users whose skills were not very advanced: novice users accounted for a $57 \%$ of these events. We provide further details on the main purposes of this tactic:

I11. To be reassured. Under a situation of uncertainty some users asked for help to be reassured: the lack of confidence or a previous disheartening negative experience led these users to employ it.

I12. To get confirmation. Some screen reader users under confusion asked about being confirmed of the current situation. For instance, u23 asked for confirmation of his current location after clicking mistakenly on a link. u23 also wanted to be confirmed whether a navigation menu was being repeated at every page in all the pages of a site.

I13. To obtain further instructions. Some novice users asked for further instructions under a variety of situations such as confusing layouts or buttons located out of the field of view of the user. Issues related to screen reader functionalities were also mentioned: users asked about activating/deactivating features, asked about more efficient reading modes when the one used was not considered effective and users also enquired when the screen reader behaved unexpectedly.

\subsection{T2: Impulsive Clicking.}

Sometimes users clicked on links without much forethought in order to escape from the problematic situation they were undergoing. This can be understood as one of the coping mechanisms employed by individuals in real life when freedom and autonomy is reduced, making individuals to focus on small gratifications [15]. In our study, users clicked on links with low information scent, which indicates that pages beyond these links were not likely to lead users towards their goals. 8 expert users employed impulsive clicking 16 times, which was implement in several ways: 
I21. Deliberately clicking on a low scented link. This happened in situations of uncertainty caused by accessibility issues, especially when pictures lacked alternative text or links were poorly labelled. Also, mobility problems such as being trapped on a loop led users to click on low scented links.

I22. Clicking on any link after coming across unexpected functionalities or content. Users would click on any reachable link in a situation of confusion produced by a previous unsuccessful exploration or a unfamiliar functionality. For instance, u20 randomly clicked on any link after landing on a page as a result of clicking on a misleading link.

I23. Clicking on any link as long as it is accessible. u11 clicked on any link that was accessible (in terms of being properly labelled) due to the uncertainty caused by surrounding inaccessible content.

\subsection{T3: Exploration Tactics.}

Exploration tactics refer to the actions taken by users to acquire more information about the environment in order to accomplish their tasks. The active exploration of issues and search for information is one of the most common coping mechanisms in real life [4]. We identified 15 users that implemented exploration tactics in several ways.

I31. Persevering. Some novice users repeated the same actions time and again, mostly under situations of confusion. These situations can be illustrated as when u21 was not able to distinguish between advertisements and search results; also, when clicking on a link did not apparently work for u22 (as he was clicking on a link that was leading him to the current page). Under these circumstances, perseverance was exhibited by 421 , who kept looking around, while u22 clicked time and again on the same link.

I32. Escaping from non-useful, non-usable or inaccessible content by tabbing down or scrolling down. This tactic was employed under situations of information overload caused by large amounts of search engine results, uncertainty caused by unexpected content and banners, and confusion produced by pieces of text that appear to be links but they are not.

I33. Fast tab/scroll/arrow down the page without completely listening to content. Users employed this tactic especially when they were certain about the location of a link within a page. This was not due to the familiarity users' might have with the page but because contents were ordered according to some criteria: in a list of alphabetically ordered countries or in a sequence of content arranged by years. The application of this technique had some risk as users took the risk of missing the content they were looking for due to a partial listening of content. It is observed that to overcome such situation recalibration and reorientation was needed at times.

I34. Moving around. Screen magnifier users explored areas of interest in a circular and outwards fashion with their screen magnifiers. Moving around is mainly employed under situations of confusion caused by unexpected banners or unclear purpose navigation menus, to name a few situations. 


\subsection{T4: Giving Up.}

Giving up is the tactic by which the user surrenders to coping. It is a last resort tactic which is employed when remaining tactics have been exhausted. In real life, surrendering to coping is a way to give up in order to lessen the perception of stress [15]. In our analysis, it was employed by 13 users due to a broad variety of causes and under different circumstances in a quite balanced way: lack of mobility accounted for 7 cases (33\%), confusion for 6 (29\%), and uncertainty and overload for 4 event each (19\%). The key situation that made users give up was not the type of problem found, as most of the problems were common to other problematic situations, but a sequence of failures and unsuccessful interactions. Therefore, the giving up tactic often occurred after navigating with difficulties through a number of pages and finding a different problem to the ones experience previously. For instance, when $u 2$ and 43 managed to escape from a loop of pages, if the next visited page contained any sort of problem, especially if it was an accessibility problem (e.g., linking pictures without alternative text or poorly labelled links), they gave up the task.

\subsection{T5: Gaining Orientation.}

We observed that users lost their orientation within a web page (local orientation) and in their traversal of different pages (global orientation). In the former case users were not able to locate themselves within a page, whereas in the latter users lost their spatial location in the sequence of pages they had traversed. 14 users employed this tactic not only to overcome mobility problems caused by disorientation as one would expect, but also to overcome confusing situations.

I51. Gaining local orientation by going to the top of the current page. Some expert screen reader users got to the top of the page after reaching the bottom of the page without finding what they were looking for or after their attention was drawn to some useless, lowly scented piece of content.

I52. Gaining global orientation by backtracking to a shelter. This tactic was employed under situations of confusion or reduced mobility. In a situation of "confusion", an example of backtracking to a shelter page happened after users clicking on links that did not lead them to complete their goals; an example of this tactic in a situation of reduced mobility was observed when users looped on some pages or when they got stuck in a dead end page.

\subsection{T6: Re-doing: Re-check, Re-type, Re-trace and Re-start.}

We observed 29 events in which 13 users repeated their actions for several reasons: to check the consistency of the outcomes in the case of re-checking, to write a more accurate keyword after getting unsatisfactory results from information repositories such as search engines or libraries, to retrace their previous unsuccessful path in order to reach a turning point and in order to start over.

I61. Re-checking. This tactic was observed on users that checked whether they had looped in a sequence of web pages; on individuals that checked twice whether the link they had clicked was actually a good choice and it was also seen on those 
users that checked whether the keyword they had typed was correctly spelled. All the tactics were employed in order to overcome confusion but in the case of loop checking, which is a situation of reduced mobility.

I62. Re-writing. A number of users rewrote their search keywords in search engines and digital libraries. Re-writing was not only employed as a technique to get better results as one would expect, but also after unsuccessfully exploring search engine results that pointed $u 4, u 6$ and $u 21$ to their actual goals; or after obtaining too many results in the case of $u 6$; keywords were also re-written after exploring a page that contained u13's goal, which was conveyed by inaccessible pictures, therefore missing this key information.

I63. Re-tracing. Some users in confusing and reduced mobility situations retraced their steps until they reached the turning point that had caused the problem.

I64. Re-starting. Some other users started over their tasks when finding themselves in a situation of reduced mobility.

\subsection{T7: Not Operating or Delegating on Assistive Technology.}

We found that 7 users employing tactics that are related to the usage (or misuse) of assistive technologies. The following tactic implementations (but I73) were mainly used under information overload circumstances.

I71. Activating screen reader functionalities. Obtaining the link list dialogue provided by JAWS, and employing functionalities that remove clutter or bypass groups of links are some of the features activated by users when too much information (textual content and links) was found.

I72. Swapping assistive technology. Depending on how well 422 could see in a given day he would employ the screen reader or the screen magnifier. However, on one occasion it was observed that due to the tiredness caused by the amount of content read out loud by the screen reader, u22 decided to stop using it and launched the screen magnifier instead.

I73. Waiting. Users stopped operating the computer when it took too long for pages to load ( 45 ) and also waited until the desired news showed while several news were looping in carousel widget (u21). Null coping was described to be one of the tactics employed by those individuals with limited autonomy [15].

\subsection{T8: Trial \& Error.}

This tactic is similar to the exploration tactic (T3) but applied to a sequence of pages instead of applying it in a single page. Users narrowed down the selection of links in their navigation towards their goal by not visiting those links that led to unsatisfactory pages. Therefore users just clicked on those links that had not been selected yet. Trial $\mathscr{E}$ error was mostly employed under situations of confusion caused by clicking on links with low information scent and when users clicked on highly scented links that did not lead them towards the goal (also called high quality distractors [2]). It was observed that after retracing (I63) and reaching the turning point of the previous failed trial, some users employed the trial $\&$ error tactic. 


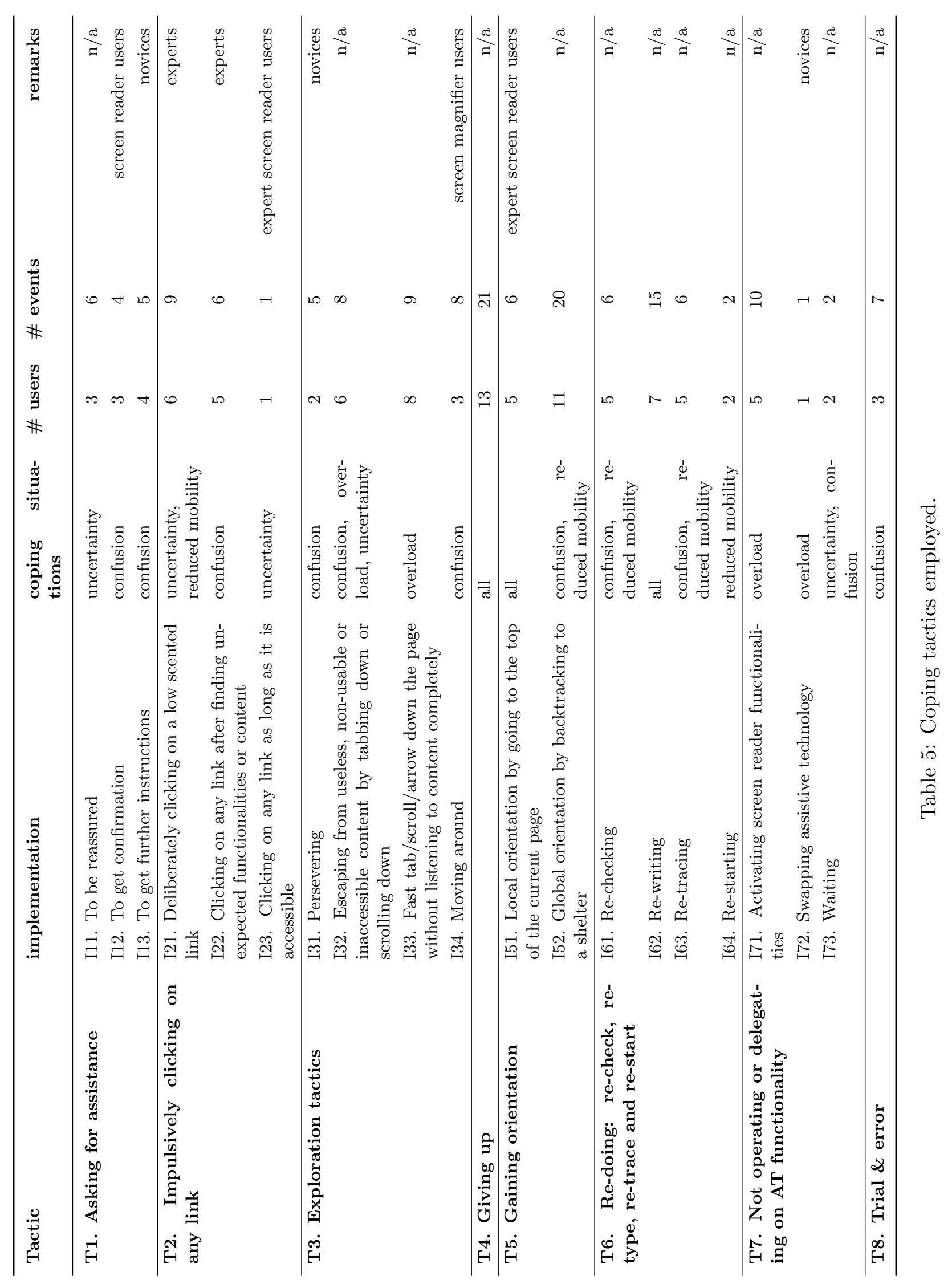




\section{Discussion}

\subsection{On the Problems and Coping Situations Discovered}

The categories found in section 4.2 are consistent with those found in literature although our categories can be considered of a higher granularity. For instance, "Content found in pages was not expected by users", "Expected functionality not present" or "Functionality does not work as expected" - a categorisation from [16] - are included in our more general "Unmet expectations".

Our analysis confirms that most of the problems encountered by visually impaired users are not caused by accessibility or usability problems. Even if the goal of this paper was not to exhaustively analyse the extent of the problems covered by existing guidelines, we found that at least those that could be considered traditional accessibility issues accounted for $18 \%$ of the problems analysed. Some of the problems belonging to the remaining categories found in section 4.2 may also be covered by accessibility guidelines. Previous research that systematically analysed guidelines coverage of problems encountered by blind users found that WCAG covered $50 \%$ of the problems [16]. Our data supports that the situation could be even worse in that the $50 \%$ would be the upper bound of accessibility problems encountered by visually disabled users which would be covered by current guidelines. It is also the case that this $18 \%$ accounts for the most problematic interactions, leaving out expected and more frequent accessibility problems.

By categorising encountered problems from a perspective of coping situations drawn from real life (see section 4.1) we were able to identify and classify coping tactics as a consequence of these problematic situations regardless of the underlying technology. For instance, should web design patterns evolve, we would expect that users who asked for help to be reassured (I11) would still be undergoing situations of uncertainty; similarly, we would expect persevering users (I31) to be subjected to confusing situations. If we wanted to ameliorate the mentioned situations independently of the underlying technology (or in addition to addressing design guidelines), providing the means to remove uncertainty and confusion would help to attenuate the problems found. In the current situation, the mapping shown in Table 1 would guide to address the general coping cases from specific situations.

\subsection{On the Tactics Employed}

In our study, we came up with 8 tactics and 17 ways in which users implemented them. These tactics can be explained in light of coping such as impulsively clicking on a link (T2), which is employed due to the lack of autonomy or freedom of the user. In this sense, the following grouping of tactics matches those circumstances that facilitate human adaptation described in section 2: keeping adequate information about the environment, maintaining satisfactory internal conditions, and maintaining autonomy and freedom:

- Exploration tactics. Exploration (T3) and trial $\&$ error (T8) are the most sophisticated tactics, as they lead the user to innovate and be more effective. In other words, T3 and T8 bring about epiphanies that provide the user with new insights and skilled behaviours. On novice users T3 and T8 are only applied 
when users already have the basic knowledge or the confidence to operate on the Web environment. The mentioned knowledge and skills are acquired through practice; otherwise, if users are not sufficiently skilled, reassurance tactics (see below) provide users with the required confidence to employ T3 and T8. Being confident seems necessary because using exploration tactics implies taking risks such as going through unexplored areas (as in I33).

- Tactics to gain reassurance. One of the observed problems is the lack of confidence at every step users make. Asking for assistance (T1) and re-doing (T6) are employed by users as techniques to reassure themselves, amongst others, by asking for confirmation about what they are doing or what is happening, by exploring what is beyond a link, and by getting feedback from their peers or from the Web environment. As a result of employing these tactics users feel more confident and gain spatial awareness about what surrounds them. Exploration tactics (see above) are often employed only if users feel in control; this situation is only reached if users have sufficient skills or if they feel confident. Therefore, it can be understood that in order to gain a certain level of autonomy, users (especially non-skilled ones) tend to get reassured by employing T1 and T6.

- Tactics to gain more freedom. Impulsive clicking (T2) and gaining orientation (T5) are employed under situations of reduced mobility, uncertainty and confusion. The usage of both techniques entails the move to a safer place where user mobility and enables users to browse without any obstacle and retake their objectives. As a last resort tactic, giving up (T4) is also considered as a tactic to gain more freedom.

As expected, tactics are not always used in isolation but are operationalised in conjunction with other tactics as a way to form strategies. For instance, in a number of occasions we observed how users went to a shelter (I52), then retraced (I63) and finally employed a trial \& error tactic (T8). We therefore understand tactics as fundamental behavioural constructs that can be composed and dovetailed to form complex strategies.

\subsection{On the Relationship between Problematic Situations and Tactics Employed}

The literature from different domains about coping suggests that individuals employ different strategies to face a given problem. Consistency in behaviours is not found in coping literature as long as the problematic situation is not very specific and concrete. This is of utmost importance because being able to establish a relationship between the tactics employed and the situations being experienced paves the way to programmatically infer problematic interactions and provide interventions to pre-empt or overcome these situations. A possible matching between emerging problematic situations and tactics employed does not allow to explicitly relate tactics to situations, while the mapping of coping situations into the tactics described in Table 2 is quite consistent. In this sense, we understand tactics as behavioural markers of cognitive processes. That is, if users decide to re-start (I64) it is likely they are undergoing a situation of reduced mobility. Similarly, if users quickly scroll down 
until reaching the bottom of the page (I33) it is very likely they are subjected to a situation of information overload. For some other tactics the phenomena occurring will not be that clear: for instance, when deliberately clicking on a low scented link (I21) users may be experiencing uncertainty or may be undergoing a situation of reduced mobility.

\subsection{Removing the Need to Cope}

In order to remove the need to cope under the situations we have uncovered one could make content accessible and usable or improve the information architecture. It is less clear how to address navigation and exploration issues, and even less clear unmet expectation, assistive technology problems or emotional coping. One possible strategy to avoid the distress caused by challenging encounters is to smooth out those situational factors that can potentially add perturbation to Web interaction: uncertainty, reduced mobility, confusion and overload. We propose a set of techniques that can help to diminish the risk of extreme adaptations:

Uncertainty. In order to avoid the uncertainty generated by web content, designing for familiarity or at least for learnability will lessen the unavoidable process of traversing through new unfamiliar grounds. On the one hand, designing for learnability mitigates the lack of skill showed by inexpert users; on the other hand, familiarity (i.e., being consistent with established practices) facilitates the use of automated interaction routines to users of a higher expertise. To do so, it is essential to promote users' understanding of the task flow, to make them aware of the functionalities of the interface and to provide help to locate and understand the goal of such functionalities [7].

Reduced mobility. Ineffective navigation strategies adopted by users lead them to getting stuck in dead-end pages and looping through pages. Some of these situations are caused by orientation problems that can be ameliorated by spatial hypermedia techniques. These techniques take advantage of the visual and spatial affordances to organise and interpret information (see the VIKI framework [14]). Similarly, Walden Paths [5] aim at addressing the temporal and linearly incremental way in which users browse by providing directed linear paths while allowing to explore off-path nodes.

Confusion. Clicking on high-quality distractors (i.e., links with strong information scent that do not lead to the goal) [2] and clicking on low scented links generates confusion on the user. When the users click on such links, they get a confusing impression as they realise that the page they land on will not lead them to the goal. Addressing such problem from a guidelines perspective (see success criteria 2.4.4 Link Purpose and 3.2.4 Consistent Identification in WCAG [3]) leads to providing meaningful texts (avoiding texts such as 'click here', 'more' and similar) and to making sure link texts are consistent: e.g., links with the same text should not link to different locations. Removing ambiguity and being consistent in link texts is crucial; however, it is a preliminary step because it does not prevent users from the problems caused by information scent or the bad execution of the re-checking technique (I61). Ideally, links should not only clearly describe the purpose of the web 
page they point to but also to provide some hints on the content users will come across. To do so, link augmentation techniques such as Gist summaries [8] that provide a summary of the page beyond a link and numeric scores of the accessibility level of the page beyond the link [20] have been proposed.

Overload. There are several factors on the web that cause information overload: high information density, the arrangement of such information and the semiserialised content rendering by screen readers. In order to remove information density some have suggested providing relevant summaries [1]; applying adaptive hypermedia techniques such as highlighting important content, hiding non-relevant content and removing clutter is another way of alleviating information overload. Some other interventions should go in line with including advanced navigation techniques in screen readers; for instance the "auto web spots" functionality of the VoiceOver screen reader allows users to navigate through areas of the web page that are visually distinguishable and meaningful.

The tactics we have identified are behavioural markers of cognitive processes that indicate problematic interactions (see table 5). Therefore, if we are able to automatically detect the employed tactics we are able to infer the sort of situations users are undergoing and provide the means to overcome them. The above techniques could be applied in these situations to alleviate or bypass extreme adaptations. We suggest that these techniques could be implemented by user agents (browsers or assistive technologies), browser add-ons or injected by proxies.

\section{Conclusion}

We have identified 17 tactics employed by visually disabled users when they experience problems on the Web. In addition to isolating the tactics we have uncovered the situations where these tactics tend to be exhibited. Consequently, not only we add a substantial amount of behavioural tactics to the existing corpus, but we also provide contextual and situational information about tactics. Relating tactics to technical problems and coping situations allows us to know what users do when they are undergoing problematic situations. This has at least two important implications when it comes to the removal of the need to cope: on the one hand, we make designers aware of the problems that bring about such situations; on the other hand, the incorporation of algorithms to detect tactics in tools will allow to infer such problematic situations in real time and provide automatic help so that users do not have to undergo extreme adaptations. These are invaluable insights for researchers and user agent developers as new research avenues on content transformation are opened.

\section{References}

[1] A. L. Berger and V. O. Mittal. Ocelot: a system for summarizing web pages. In Proceedings of the 23rd annual international ACM SIGIR Conference on 
Research and Development in Information Retrieval, SIGIR'00, pages 144-151, New York, NY, USA, 2000. ACM.

[2] D. Brumby and A. Howes. Strategies for guiding interactive search: An empirical investigation into the consequences of label relevance for assessment and selection. Human-Computer Interaction, 23(1):1-46, 2008.

[3] B. Caldwell, M. Cooper, L. G. Reid, and G. Vanderheiden. Web content accessibility guidelines (wcag) 2.0. World Wide Web Consortium, Dec. 2008.

[4] G. Caplan. Principles of Preventive Psychiatry. Basic Books, 1964.

[5] R. Furuta, F. Shipman, C. Marshall, D. Brenner, and H.-W. Hsieh. Hypertext paths and the world-wide web: experiences with walden's paths. In Proceedings of the 8th ACM Conference on Hypertext, HYPERTEXT'97, pages 167-176. ACM, 1997.

[6] B. Glasser and A. Strauss. The Discovery of Grounded Theory: Strategies for qualitative research. Aldine, 1967.

[7] T. Grossman, G. Fitzmaurice, and R. Attar. A survey of software learnability: Metrics, methodologies and guidelines. In Proceedings of the 27 th International Conference on Human Factors in Computing Systems, CHI'09, pages 649-658, New York, NY, USA, 2009. ACM.

[8] S. Harper and N. Patel. Gist summaries for visually impaired surfers. In Proceedings of the 7th international ACM SIGACCESS Conference on Computers and Accessibility, Assets'05, pages 90-97, New York, NY, USA, 2005. ACM.

[9] R. Lazarus and S. Folkman. Stress, Appraisal, and Coping. Springer, 1984.

[10] R. Lipshitz and O. Strauss. Coping with uncertainty: A naturalistic decisionmaking analysis. Organizational Behavior and Human Decision Processes, 69(2):149-163, 1997.

[11] D. Lunn. Verification of the coping strategy framework through an analysis of the nova evaluation data. July 2008.

[12] D. Lunn and E. Michailidou. Observational notes acquired from henshaws' skillstep to success class: Observation period 1. Technical Report SADIe Technical Report 5, The University of Manchester, School of Computer Science, July 2007.

[13] D. Lunn and E. Michailidou. Observational notes acquired from henshaws' skillstep to success class: Observation period 2. Technical Report SADIe Technical Report 8, The University of Manchester, School of Computer Science, February 2008.

[14] C. Marshall and F. Shipman. Spatial hypertext: designing for change. Communications of ACM, 38(8):88-97, August 1995. 
[15] R. Moos. Human Adaptation: Coping with Life Crises. Heath, 1976.

[16] C. Power, A. Freire, H. Petrie, and D. Swallow. Guidelines are only half of the story: accessibility problems encountered by blind users on the web. In Proceedings of the 2012 ACM annual conference on Human Factors in Computing Systems, CHI '12, pages 433-442, New York, NY, USA, 2012. ACM.

[17] R. Savolainen. Filtering and withdrawing: strategies for coping with information overload in everyday contexts. Journal of Information Science, 33(5):611$621,2007$.

[18] M. Vigo and S. Harper. Human adaptation as a behavioural strategy: Preliminary considerations for web interaction. Technical report, August 2011.

[19] M. Vigo and S. Harper. Human adaptation to the web: A proposal for a research programme. Technical report, September 2011.

[20] M. Vigo, B. Leporini, and F. Paternò. Enriching web information scent for blind users. In Proceedings of the 11th international ACM SIGACCESS conference on Computers and accessibility, Assets '09, pages 123-130, New York, NY, USA, 2009. ACM.

[21] R. White. Strategies of adaptation: An attempt at systematic description. In Coping and Adaptation. Basic Books, 1974.

[22] Y. Yesilada, R. Stevens, S. Harper, and C. Goble. Evaluating dante: Semantic transcoding for visually disabled users. ACM Transactions on ComputerHuman Interaction, 14(3), Sept. 2007. 


\section{Associated Files}

